\title{
Contrast Analysis in Latex/Surfactant Aqueous Suspensions
}

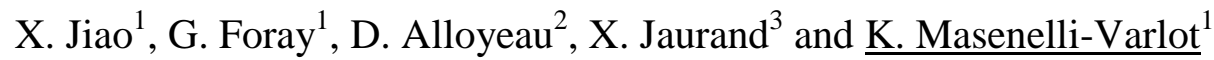 \\ ${ }^{1 .}$ Université Lyon, INSA-Lyon, UCBL, MATEIS, CNRS UMR 5510, F-69621 Villeurbanne, \\ France. \\ 2. Laboratoire Matériaux et Phénomènes Quantiques, Université Paris Diderot - CNRS, Paris, \\ France. \\ 3. Université Lyon, Université Lyon 1, CT $\mu$, F-69622 Villeurbanne Cedex, France.
}

Several systems are now commercially available to analyze samples in liquid. They can be classified into two main categories. The first one corresponds to liquid cells, where the liquid is encapsulated into two thin membranes. The cell is then introduced into a Transmission Electron Microscope (TEM) operating under vacuum. Eventually, the liquid can flow or may be heated, which requires specific sample holders. If no heating or flow is required, newly designed cells can even be placed onto regular sample holders. The second category corresponds to Environmental Scanning Electron Microscopes (ESEM). When cooled down to a few degrees and when surrounded by the appropriate gas pressure, a liquid droplet can be maintained at the liquid-vapor equilibrium. In all cases, a well suited imaging mode corresponds to the collection of scattered electrons in transmission, which gives rise to a mass-thickness contrast [1].

As these systems use very different experimental parameters (sealed cell or dynamic equilibrium, low or high acceleration voltage, among other parameters), we aimed at comparing the contrasts obtained and determine in what extend such systems can provide complementary information. To do so, we used an aqueous suspension containing SBA-PMMA particles of diameter around $200 \mathrm{~nm}$ (BASF R\&D Lab) and a high molecular weight surfactant combining steric and ionic functions (XPCAS 803 from PCAS, Longjumeau, France). Three different systems were tested : a homemade device fitting onto an ESEM stage [2], a commercial liquid cell sample holder (Poseidon sample holder from Protochips) and commercial sealed cells fitting onto conventional TEM sample holders (K-kit cells from Bio Ma-Tek).

Figure 1 gives typical images acquired in the ESEM or with the liquid cell sample holder on the suspension containing latex particles and surfactant. It is obvious that the spatial resolution is far better in TEM than in ESEM, as individual surfactant molecules can be distinguished in TEM around the latex particles. The measures of the spatial resolution will be compared with theoretical values calculated from the literature. More interestingly, the contrasts between the water layer, the latex particles and the surfactant molecules are very similar and do not seem to depend significantly on the acceleration voltage. Intensity profiles will be compared with theoretical ones obtained through Monte Carlo simulations in order to decouple the effects of the acceleration voltage and of the water film thickness. Finally, the visible changes induced by irradiation damage, such as morphological changes or contrast changes during time, will be discussed in light of the literature on both electron-liquid interactions and irradiation damage in polymers. [3]

\section{References:}

[1] N de Jonge and FM Ross, Nat Nanotechnol 6 (2011), p. 695.

[2] A Bogner et al, Ultramicrosc 104 (2005), p. 290.

[3] The authors acknowledge the Consortium Lyon Saint-Etienne de Microscopie (CLYM) for the access to the microscopes and the company LFG for having kindly provided the K-kit cells. They also acknowledge the China Scholarship Council (CSC), Institut Universitaire de France (IUF) and the CNRS network "Microscopie Electronique et Sonde Atomique" (METSA, FR CNRS 3507) for financial support. 
a)

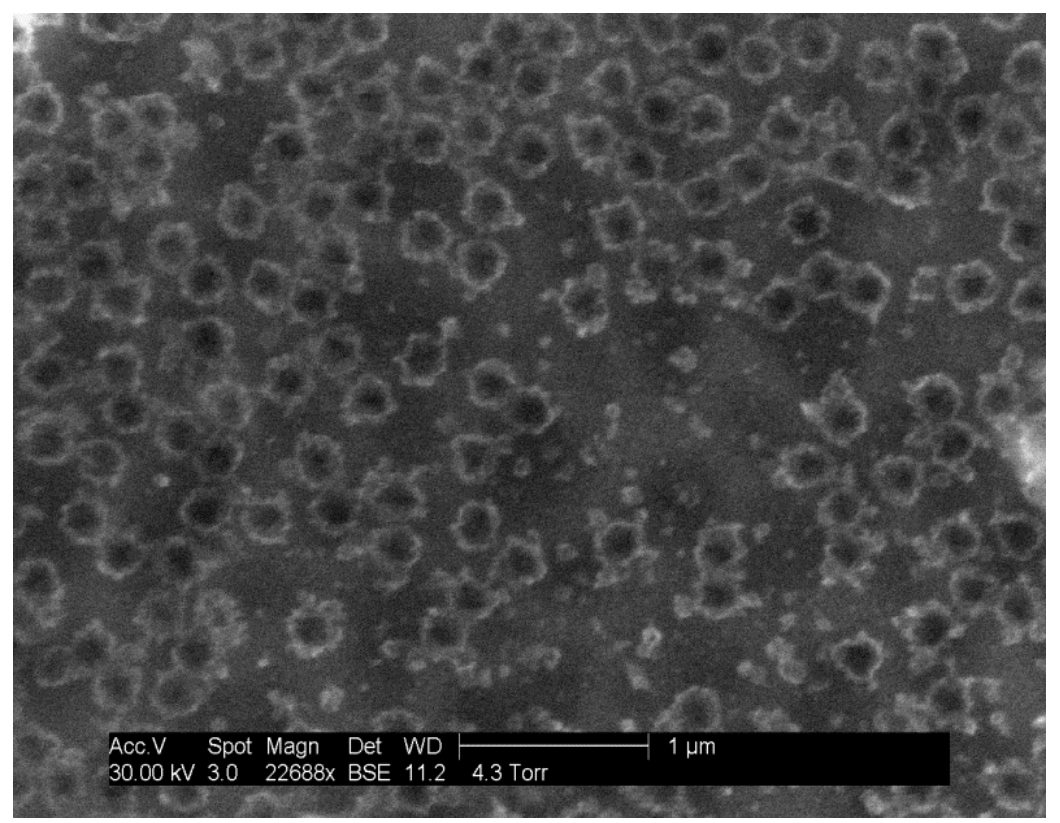

b)

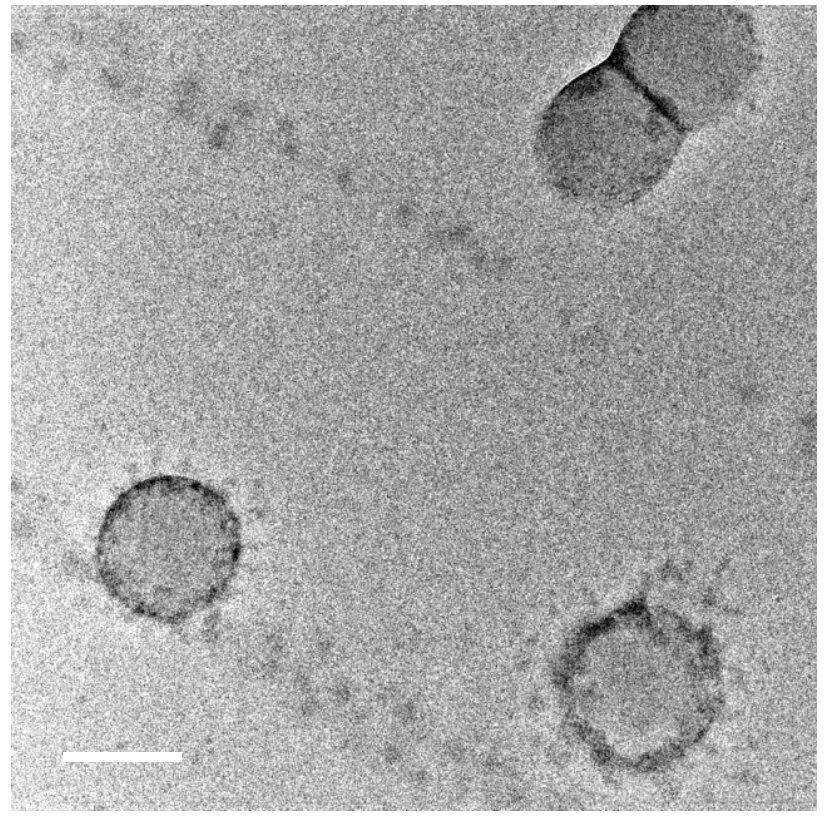

Figure 1. Images obtained on a sample containing SBA-PMMA latex particles and a surfactant.

a) Image obtained at $30 \mathrm{kV}$ using an ESEM, on a droplet of liquid deposited on a holey carbon film. Dark field mode; b) Image obtained at $200 \mathrm{kV}$ using a TEM with a droplet of liquid encapsulated between two silicon nitride membranes. Bright field mode. 